\title{
Factors influencing diabetes self-management among Omani patients with type-2 diabetes: Patients' perspectives
}

\author{
Hilal H. Alrahbi ${ }^{* 1}$, Said A. Alghenaimi ${ }^{2}$ \\ ${ }^{1}$ Diwan of Royal Court Medical Services. Muscat, Oman \\ ${ }^{2}$ Rustaq Nursing Institute, Ministry of Health, Rustaq, Oman
}

Received: June 19, 2017

DOI: $10.5430 /$ jnep.v7n12p64

\author{
Accepted: July 20, 2017 \\ Online Published: July 23, 2017 \\ URL: https://doi.org/10.5430/jnep.v7n12p64
}

\begin{abstract}
Background and aim: Management of diabetes requires using different approaches that include self-management in which the patients are the main key players. Diabetes self-management contributes to preventing diabetes-related complications and improving the QOL of patients with diabetes. The aim of this study was to explore the factors influencing diabetes selfmanagement from the perspectives of the patients with type-2 diabetes in Oman.

Methods: A qualitative descriptive design using semi-structured individual interviews was used to conduct this study. A purposive sample of 21 Omani patients with type-2 diabetes were interviewed. Interviews were transcribed verbatim and translated into English. Content analysis and constant comparison were used to analyze the data.

Results: Eight factors influencing DSM were identified: awareness of diabetes complications and the importance of DSM, ability to adapt, support, fear of consequences, frustration and helplessness, complying with sociocultural norm/ritual, lack of care, and planning.

Conclusions: To improve the quality of diabetes care and decrease the devastating diabetes-related complications, managing diabetes has to take the form of a collaborative approach that put into consideration the factors influencing diabetes selfmanagement that were identified by this study.
\end{abstract}

Key Words: Nurses, Oman, Qualitative, Self-care, Self-management, Type-2 diabetes

\section{INTRODUCTION}

Diabetes mellitus represents a health burden at the global and individual levels. At least $12 \%$ of the total healthcare expenditures in many countries are spent in managing diabetes and diabetes-related complications. Every six seconds someone dies from diabetes, accounting for approximately death of 5.0 million people aged between 20 and 79 years in 2015. ${ }^{[1]}$ At the individual level, diabetes requires a high level of responsibility. Individuals with diabetes need to adjust to a new lifestyle that involves commitment to a diabetic diet regimen, exercise, use of medications, blood glucose monitoring, and foot care ${ }^{[2]}$ Further, a diagnosis of diabetes carries its own psychological and social burdens. For example, depression accounts for $10 \%$ to $30 \%$ among patients diagnosed with diabetes. ${ }^{[3]}$

Management of diabetes takes the form of multidimensional approaches. The main goal of diabetes treatment is glycemic control to prevent or delay the development of diabetesrelated complications. A collaborative plan of treatment

*Correspondence: Hilal H. Alrahbi; Email: arhab141@ hotmail.com; Address: Diwan of Royal Court Medical Services, Muscat, Oman. 
that positions the individuals with diabetes in the center of the management plan to assume roles in their care is crucial, and mainly occurs through diabetes self-management (DSM).${ }^{[4,5]}$ Hence, DSM is considered an integral part of diabetes management. It contributes to preventing and lessening the severity of long-term diabetes-related complications and improving the QOL of patients with diabetes. ${ }^{[5,6]}$ Nonetheless, DSM is a complex lifelong journey that requires commitment, specific skills, knowledge, and confidence. ${ }^{[7]}$

Diabetes mellitus is recognized as a major health issue in Oman. ${ }^{[8,9]}$ Oman is located in the southeastern quarter of the Arabian Peninsula. Administratively, Oman is divided into 11 governorates (provinces). ${ }^{[10]}$ The healthcare service in Oman is free and is mainly regulated, delivered, and maintained by the Ministry of Health $(\mathrm{MOH})$ through a national health care system. According to the IDF, ${ }^{[1]}$ it was estimated that the prevalence of diabetes mellitus in Oman in 2015 was $14 \%$. National surveys conducted between 1991 and 2010 in Oman indicated that the age-adjusted prevalence of type- 2 diabetes in Oman varied from $10.4 \%$ to $21.1 \% .^{[9]}$ The prevalence of diabetes-related complications such as retinopathy ${ }^{[11,12]}$ and microalbuminurea ${ }^{[13]}$ are escalating. Many patients with diabetes lack awareness and knowledge of diabetes and its complications. ${ }^{[14,15]}$ Research has indicated that diabetes care is suboptimal, ${ }^{[16,17]}$ and hindered by many challenges such as lack of human resources, medications, and laboratory support. ${ }^{[17]} \mathrm{A}$ key element in improving the quality of diabetes care recognized by many researchers in Oman ${ }^{[16-18]}$ was through implementing self-management programs.

Although previous research has indicated that DSM is suboptimal among Omani patients, ${ }^{[15,19]}$ and that DSM is considered the cornerstone of diabetes management, ${ }^{[5,20]}$ no study has investigated the perspectives of Omani patients with type2 diabetes about factors influencing self-management. The current study aimed to fill this knowledge gap and explore such factors. Understanding such factors is crucial in managing diabetes because it is necessary for the development of appropriate interventions to improve the quality life of patients with diabetes, prevent complications, and subsequently decrease the associated financial and personal costs.

\section{Methods}

\subsection{Design}

A qualitative descriptive design using semi-structured individual interviews was used to conduct this study. ${ }^{[1]}$

\subsection{Sample}

A purposive sample of 21 Omani patients with type- 2 diabetes was recruited to participate in this study. Using a

Published by Sciedu Press maximum variation sampling strategy, the researchers purposefully selected the participants based on their personal judgment about which participants were more typical or productive to the study purpose. ${ }^{[22]}$

Inclusion criteria included being diagnosed with type-2 diabetes for at least 2 years, cognitively and physically competent, aged 20 years or above, able to speak and understand Arabic language, and willing to participate in the study.

\subsection{Setting}

Recruitment was conducted in the diabetes clinic of the Diwan of Royal Court Polyclinic; a governmental healthcare institution located in the capital of Oman, Muscat. This polyclinic provides primary healthcare services to the employees of the Diwan of Royal Court and their families who come from all over the country.

\subsection{Data collection}

Data was collected between November 2015 and May 2016. Participants were approached on the day of their appointments and invited to voluntarily participate in the study. A total of 35 patients initially interviewed, however, 14 of them refused participation because they felt uncomfortable to be audio recorded. Their data was discarded and was not included in the study findings. Data saturation was reached after 21 interviews, which were recorded using two digital sound recorders. Interviews were conducted in Arabic by the researchers and one research assistant, which were then transcribed verbatim and translated into English by a proficient interpreter.

Each semi-structured individual interview lasted between 5075 minutes. All interviews took place in one of the clinic's rooms that was comfortable and well ventilated. Along with the open-ended questions, a topic guide was used to focus the discussion on the participants' perceptions about the factors influencing DSM. Probes questions were used to clarify or elicit additional responses from the participants. ${ }^{[23]}$ To achieve accurate and comprehensive data collection, handwritten notes were taken by the researches to capture any new ideas that emerged during the interviews. ${ }^{[24]}$ To ensure consistency and rigor in data collection, the researchers and the research assistance met prior to the commencement of data collection during which the topic guide was discussed to reach a common understanding among all. In addition, the researchers and research assistant conducted the first interview together followed by a debriefing session that aided in clarifying doubts pertaining to understanding of the topic guide. 


\subsection{Data analysis}

Content analysis and constant comparison were used to analyze the data. ${ }^{[25]}$ The researchers read and reread the interview transcripts, coded them, identified major categories that were divided into segments, searched for themes, and closely compared them to each other for similarities, commonalities, recurring regularities, and relationships within the categories of the data.

\subsection{Ethical consideration}

This study was approved by the Research and Ethical Review and Approve Committee (RERAC) of the MOH-Oman (Ref.MH/DGP/R\&S/PROPOSALAPPROVED/26/2014). Informed written consent was obtained from each participant prior to the interviews. All interviews were conducted on the same day the consent was taken due to the fact that these patients come from different regions of the Oman for treatment at this out-patient polyclinic, and that access to them will be a challenge if they sign up to participate for a different day. Participation was voluntary. All interviews were identified by codes and anonymity was assured.

\section{Findings}

A total of 21 semi-structured one-to-one interviews were conducted. Majority of the participants $(71.4 \%)$ were male, and most of them (57\%) aged between 40-49 years. Most of them were diagnosed with diabetes between 2-6 years (see Table 1).

Table 1. Demographic Characteristics $(\mathrm{N}=21)$

\begin{tabular}{llll}
\hline Variables & Frequency & Variables & Frequency \\
\hline Gender & 15 & Marital status & 20 \\
Male & 6 & Married & 1 \\
Female & & Not married & 8 \\
Age & 3 & Level of education & 8 \\
$30-39$ years & 12 & Middle school & 5 \\
$40-49$ years & 6 & High school & 18 \\
$50-59$ yearsP & & College level & 3 \\
Employment & 17 & Type of medication & 10 \\
Employed & 4 & Pills only & 2 \\
Not employed & & Pills with insulin & 5 \\
Duration of DM (years) & 12 & Region & Muscat \\
$1-5$ & 3 & Aldhakiliyah & \\
$6-10$ & 4 & Batina & \\
\hline 11-20 & 2 & & \\
\hline
\end{tabular}

Eight factors influencing DSM were identified: awareness of diabetes complications and the importance of DSM, ability to adapt, support, fear of consequences, frustration and helplessness, complying with sociocultural norm/ritual, lack of care, and planning. Provided below are detailed descriptions of each factor supported with excerpts from participants.

\subsection{Awareness of diabetes complications and the impor- tance of DSM}

Despite the fact that each participant had at least one diabetes education session, a dominant factor discussed throughout the interviews was lack of awareness of diabetes, its complications, and the importance of DSM. Many participants indicated that more attention should be paid to diabetes selfmanagement education (DSME), and that the amount of time currently spent educating patients is not sufficient. They also indicated that the contents are broad and taught in a didactic method over a short period of time, which makes it challenging to patients to comprehend them. They aspire to see technology such as smartphone applications used to teach DSM. Some participants indicated that they fail to make a connection between DSM and diabetes control because the emphasis in most of the time is drawn to the importance of taking tablets.

"I believe I want more understanding of my disease. It is very complex to me to process everything said about diabetes and other things related to it in one or two short sessions. I understand that doctors and nurses are very busy, but I really want to reduce the trips to the hospital if 
I can learn more about the care of myself."

"Nurses told me so many things and gave me many papers about diabetes, I feel there should be a better way to educate patients so it stays in my mind; may be a video or an interactive thing, something like that."

\subsection{Ability to adapt}

Many participants expressed powerless to adjust to live with diabetes. They indicated that the diagnosis with diabetes comes with stress and changes in their life style. Many compromises have to be done, particularly when it to comes to diet, sleep time, and exercising. This has negatively affected their adherence to DSM.

"It is not easy to be a diabetic. Your life gets disturbed; sometimes I get exhausted because of the many things I have to do."

Other participants believed in faith to adjust to diabetes. Strong believe in Allah (God) has helped them to perceive diabetes as a blessing from Allah that would qualify them to gain more good deeds and earn heaven hereafter.

"I am a very strong believer in Allah. Nothing happen in this life, whether good or bad without Allah willing. I am satisfied with anything that comes from Allah. My disease is from Allah, and I should never question his willing."

\subsection{Support}

Support or lack of support from individuals such as healthcare providers, family members, and/or friends was reported by many participants to be one of the factors affecting DSM. Some participants stated that a family member such as a spouse has helped them in day-to-day management of diabetes. Taking part in the house chores, reminder about medications and blood glucose test, and going for a walk with them were perceived as crucial spousal support to deal with diabetes. One participant reported that sometimes her family supports her by cooking a meal for the whole family that has no/less sugar. Others reported that hearing something like "good job" from a family member or a friend motivated them to continue on their healthy diet and exercise.

"Diabetes is really a tiring disease. Without my husband's help, I would have given up."

"One of my friends goes with me for a walk around the neighborhood 4 times a week. We do this after almagrib (dusk) prayer for about 30-40 minutes. I feel comfortable that someone is encouraging me to stay healthy."

Published by Sciedu Press
Support from healthcare providers such as nurses and doctors was also expressed by participants as one of the factors motivated them to manage diabetes independently. They indicated that some doctors and nurses are very welcoming and spend enough time with patients to explain about the management plan. However, others reported that some HCPs pay less attention to their patients during clinic visit. Such providers spent more time on the computer writing their medical notes, prescribing medications etc.

"I really feel sense of caring when my doctor and my nurse ask more questions about me and how I am living with diabetes."

\subsection{Fear of consequences}

Fear of the consequences of diabetes-related complications such as amputation, blindness, and death was described by the majority of participants as a motive to adhere to DSM. Participants were very worried about losing their roles in life as a result of such complications. It was expressed that adhering to DSM is a responsibility not only toward themselves but most importantly toward their families.

"I am so worried; I don't want to lose my leg because of my negligence. I have 5 kids, and I feel responsibility toward them. No one would take care of them if I become handicapped due to diabetes. I must stick to what the doctor tells me to do."

\subsection{Comply with sociocultural norms/rituals}

Complying with sociocultural norms or rituals was identified by many of the participants as a factor that influenced DSM. As per the cultural norms in Oman, people gather frequently in different occasions at the level of the family, the neighbors, and the whole villagers. It is not uncommon that you will be invited for a "kahwa" (Omani traditional banquet comprises of dates, fruits, and coffee) while you are taking a walk around the neighborhood, shopping from the nearest grocery, or after praying at the nearest mosque. Although this invitation may be easily disregarded in some culture, doing so in Oman could be considered as an offense. People in Oman are socially connected by virtue of hospitality, which was identified by many of the participants as an important factor that influenced their social relationship. Complying with such sociocultural norms or rituals has challenged some participants to follow their diet control. They expressed being embarrassed at social events due to their diet restrictions.

"You understand, we are Omani, and it is very difficult to refuse their invitation. I cannot say no to people when they offer me food at their 
homes that has high sugar. I don't want to offend them."

Other participants indicated that they were less challenged when offered unhealthy food by a friend or a family member.

"I have no problem telling people around me about my diabetes. I believe they should know so they would not insist to offer me food with so much of sugar. My only problem is when I am invited to an event outside the family gathering in which accommodating my food choices would be difficult."

Additionally, many female participants expressed being embarrassed to exercise alone without a "mahram" "woman' closest male relative and her guardian, usually her father or husband, or someone to whom the woman could not be legally married".[26] In many situations, they had to skip going for a walk around the neighborhood because a mahram was not available.

"I feel shy walking alone in the neighborhood. It is the ritual. I have to wait for my husband to do that. When he is not there, I either ask one of my sons or sometime my sister. But I walk with my sister only at night; and she is not there always for me."

\subsection{Frustration and helplessness}

Feeling frustration and helplessness from not having good results contributed to lack of DSM among participants. In spite of adherence to DSM, lack of glycemic control along with experiencing some of the complications of diabetes made participants quit or question the value of adherence to DSM activities.

"I don't think I can do anything about it. Regardless of what I do, the glucose in my blood remains high. I do walk 30 minutes, 5 days a week. I feel what is going to happen is going to happen."

\subsection{Lack of care}

Some participants indicated that there is a lack of sufficient number of qualified diabetologists, podiatrists, and diabetes educators available in the area they live which hinders the quality of care they receive. They expressed frustration regarding longer waiting time on the day of their appointment, particularly if they had to see another HCP such as the dietitian, podiatrist, and/or diabetes educator. Participants were also unhappy by the fact that they see different physician every time they go for their appointment. They preferred getting treated by the same physician because it gave them the sense of individualized care and was perceived as important in the continuity of their care.

"I believe the government should provide more qualified healthcare providers in all regions of Oman. I live in a small village, $150 \mathrm{~km}$ from here. Not everyone has a car like me to drive to this health center."

Although access to healthcare services in Oman is free, most of the equipment needed for self-care outside the hospital are not covered. Therefore, due to the high cost, some participants were unable to buy a glucometer and/or glucose strips to self-monitor their blood glucose at home. They reported that their blood glucose goes unchecked for weeks.

"In most of the time I come to the clinic, I see a different doctor. I have to tell the whole history of my disease again. I really like to see Dr. ,„. She understands my concerns more than other doctors."

\subsection{Planning}

Good or lack of planning has been reported by many participants to influence DSM. Time constraints and inability to balance between work, home, children, and social commitments were among the identified issues that renders participants to skip exercising and/or checking blood glucose. Disorganization of the day schedule causes some participants to forget taking medication in the prescribed time.

"I admit, there is time, but it not as easy as it sounds. I have to be very organized. Some days you are so tired and you forget to do many things you are supposed to do for your disease."

\section{Discussion}

This study presented many factors that influenced DSM from the perspectives of the patients with type- 2 diabetes. Although research in Oman ${ }^{[15,19]}$ has indicated that DSM among the patients is suboptimal, factors influencing DSM from the perspectives of the patients have never been explored.

Awareness of diabetes complications and the importance of DSM was one of the factors discussed by the participants. The discussion focused on the impact of DSME in changing the perspective of the patients toward diabetes self-care. Lack of knowledge and awareness about DSM causes patients to less adhere to DSM as several studies in Oman ${ }^{[14,15,27]}$ and elsewhere ${ }^{[6,28]}$ have indicated. Through DSME, patients become equipped with the needed knowledge and skills that make them independent in the care of their disease. ${ }^{[6]}$ Participants in this study view the current DSME as deficient, and it 
does not meet their intellectual and cultural needs. ${ }^{[29]}$ Many of the educational contents are dense, lack simplicity, and lacks examples from the Omani culture such as pictures of traditional food. Participants, particularly those with higher educational attainments, preferred non-traditional way of delivering educational contents such as using smartphone applications. Comprehending contents about diabetes and DSM is complex; it requires time and using variety of teaching strategies. ${ }^{[7]}$ For such patients to integrate DSM into their lifestyle, enough attention should be paid to educating them.

Consistent with other studies, ${ }^{[3,28]}$ the participants in this study expressed challenges dealing with and adjusting to their disease. The diagnosis with diabetes brings stress and depression to the life of these patients. ${ }^{[3]}$ Many lifestyle modifications and commitments need to be taken by such patients particularly to diet and exercising. Failure to adapt to the new routines of living with diabetes renders patients dependent and less adhere to DSM. Healthcare providers need to be aware of the alarming signs of maladaptation to diabetes so as proactive measures may be taken to improve the quality of life of patients with diabetes.

An important element to effective DSM is having support from family, friends, and healthcare providers. ${ }^{[30]}$ Likewise, our participants identified support as a crucial part in helping them in self-management and integrating diabetes into their lives. Through the encouragement from a family member, friend, and/or healthcare provider, participants felt motivated to adhere to the DSM practices. Support, specifically from family and friends, represented in helping the participants in dealing with diabetes issues encountered on day-to-day bases such as hypoglycemia, checking blood glucose, preparing healthy meal, and accompanying them for hospital appointments. Such support promotes patient's sense of control and empowers them to manage their diabetes care independently. ${ }^{[28,30]}$ Similarly, healthcare providers are instrumental in supporting their patients to care for their diabetes. ${ }^{[30]} \mathrm{Un}$ fortunately, many participants felt that healthcare providers paid less attention to the issue of patient support. Among the issues that distracted healthcare providers from fully attending to patients during hospital visits are filing medical records, responding to telephone calls, and talking to other healthcare providers. A fundamental role of healthcare providers is to promote a collaborative supportive environment to help patients live with diabetes and improve quality of care. ${ }^{[7,31]}$ Further, healthcare provider need to efficiently utilize information technology such as smartphone applications to remotely monitor patients adherence to DSM will allow the patients to be actively involved in their care and would promote patient centered care approach.
Fear of consequences of diabetes has also emerged as an important factor affecting self-management. Consistently, research has indicated that fear of losing role due to diabetes complications such as amputation or blindness motivated patients to adhere to diabetes regimen and DSM. ${ }^{[32]}$ Responsibility toward family was one of the major elements participants identified as motive to stay healthy and care for their diabetes. This is particularly correct with a culture such as Oman where family is the essence of life. Taking care of family is derived by religious and cultural obligations, and failure to comply with such obligations accounts for social stigma. Healthcare providers should discuss diabetes complications frequently with their patients to make them aware of how devastating such complications can be. Family and friends may also be made aware of diabetes complications as their supportive role cannot overemphasize.

The culture in which patients with diabetes live has an impact on adherence to self-management activities. ${ }^{[31]}$ Many participants in the current study were challenged by complying with the sociocultural norms and rituals to constantly practice DSM activities. For example, some of them had to accept eating high carbohydrate food during social events to avoid offending their invitees. Likewise, observing religious and cultural rules deter some women from exercising in public. Understanding cultural norms and rituals makes healthcare providers in a better position to intervene accordingly. $\mathrm{Pa}-$ tients should learn how to appropriately overcome situations of embarrassment such as when attending social gathering. They need to be empowered to set forth rules that stop them from compromising their health. As recommended in previous research, ${ }^{[18]}$ making gymnasiums available for women throughout the country would help them stay physically active.

Comparable to our findings, several research indicated that frustration and helplessness with success in achieving optimal levels of glycemic control discouraged patients from practicing DSM activities regularly. ${ }^{[28,33,34]}$ Such feelings cause depression to some patients and consequently make them neglect caring for their diabetes. ${ }^{[28]}$ Healthcare provider's role goes beyond conducting generic technical assessment for glycemic control or diabetes complications. Screening for signs of depression or ineffective copying should be an integral part in the management plan of diabetes. ${ }^{[35]}$ Patients should be encouraged to freely express themselves and discuss any worrying symptoms that potentially may lead to feeling of frustration and/or helplessness.

Further, the participants in this study reported lack of available resources in the area they live such as available qualified diabetologists, podiatrists, diabetic nurses, and diabetes edu- 
cators in the area they live. While the healthcare services in Oman have witnessed tremendous improvement, the quality of care of diabetes has been reported to be sub-optimal. ${ }^{[16-18]}$ It is needless to say that improvement in the quality of care of diabetes contributes to preventing the devastating complications of diabetes mellitus and thereby reducing the burden on the patients, their families, and the healthcare cost. ${ }^{[5,6,36]}$ Decision makers should ensure that enough resources are made available for the care of patients with diabetes.

A fundamental issue in the process of self-management of diabetes is good planning and organization. ${ }^{[30]}$ Many patients, like in the current study, failed to balance between home, work, and caring for their diabetes. Being regular on exercise, self-monitoring of blood glucose, and taking medication, along with dealing with other issues in life challenged the participants to stay focused and organized. Healthcare providers need to teach patients different planning strategies such as using a planner, mobile applications, and/or a reminder when possible, so that they become independent and confident in organizing their day-to-day or weekly activities.

\section{Limitations of the study}

The findings of this study should be interpreted in light of its limitations. Similar to many qualitative studies, the sample was small, purposive, and represents few numbers of regions of Oman. The findings of this study might have not capture the perspectives of patients in other regions. Most of the participants were males and this might have not presented enough gender-mixed views. While quantitative approach is the best method to quantifying the factors affecting DSM, the strength of this study lays in its ability to capture broader perspectives of such factors from the patients' standpoints.

\section{Conclusion}

Diabetes self-management is considered to be an integral part of diabetes management. While that is true, the process of self-management is complex and requires that healthcare providers understand how patients are managing their diabetes outside the healthcare setting. To the best knowledge of the researchers, this study was the first to explore the factors influencing the process of diabetes self-management among Omani patient with type- 2 diabetes. It has presented new knowledge in the area of diabetes self-management which can be used to improve the quality of diabetes care in Oman. Identifying such factors is crucial for healthcare providers, researchers, educators, and decision makers. When planning the care for patients with diabetes, these factors need to be considered. For example, patients need to be educated about their diabetes using different teaching methods and in a way that meets their needs, so that they become autonomous and integral participants in their care. Further, anticipatory assessment of patients' feelings, challenges, and motives that impact adherence to DSM should be done by healthcare providers during the care of patients with diabetes. Based on the findings of this study, further research in the area of DSM in Oman needs to be conducted to improve the quality of diabetes care. The factors presented by this study may be further explored and their relationship to promote DSM may be tested in further study such as the use of smartphones to promote adherence to DSM. Hence, different DSME strategies targeted to facilitate adherence to DSM may be established.

\section{ACKNOWLEDGeMENTS}

The researchers would like to acknowledge the Directorate General of Medical Services, Diwan of Royal Court, Oman for the in-kind support this study has received. From the same organization, we also acknowledge the head and the staff of the department of Internal Medicine where the study took place for their support in making the data collection process easier. Finally we acknowledge Mr. Ahmed Alrahbi, nurse specialist for his assistance in data collection.

\section{CONFLicts OF InTEREST Disclosure}

The authors declare no conflict of interest.

\section{REFERENCES}

[1] International Diabetes Federation (IDF). IDF diabetes atlas-2015 2015 [cited 2017, March 15]. Available from: http://www.diab etesatlas.org

[2] American Diabetes Association. 6. Glycemic targets. Diabetes care. 2015 Jan 1; 38(Supplement 1): S33-40. https ://doi.org/10.2 337/dc15-S009

[3] Roy T, Lloyd CE. Epidemiology of depression and diabetes: a systematic review. Journal of Affective Disorders. 2012; 142: 8-21.

[4] American Diabetes Association (ADA). Standards of medical care in diabetes-2015. Diabetes Care. 2015; 38(1): 1-2. https://doi.or $\mathrm{g} / 10.2337 / \mathrm{dc} 15-\mathrm{S} 001$

[5] Powers MA, Bardsley J, Cypress M, et al. Diabetes self-management education and support in type 2 diabetes a joint position statement of the American Diabetes Association, the American Association of Diabetes Educators, and the Academy of Nutrition and Dietetics. The Diabetes Educator. 2015; 41(4): 417-30. PMid:26047627 https://doi.org/10.1177/0145721715588904

[6] Lorig K, Ritter PL, Villa FJ, et al. Community-based peer-led diabetes self-management a randomized trial. The Diabetes Educator 2009; 35(4): 641-51. https://doi.org/10.1177/0145721709 335006 
[7] McBain HB, Mulligan K, Haddad M, et al. Diabetes self-management in people with severe mental illness. The European Health Psychologist. 2014; 16(6): 240-8.

[8] Alwan A, Jarrett J. Review of the National Diabetes Programme in Oman. Geneva: World Health Organization; 2002.

[9] Al-Lawati JA, Panduranga P, Al-Shaikh HA, et al. Epidemiology of Diabetes Mellitus in Oman: Results from two decades of research. Sultan Qaboos University Medical Journal. 2015; 15(2): 226-33.

[10] Ministry of Tourism (MOT). Oman geography and history 2016 [cited 2017, Feb. 5]. Available from: http://www . omantourism. gov.om

[11] Khandekar RB, Mohammed AJ. Visual disabilities among diabetics in Oman. Saudi Medical Journal. 2005; 26(5): 836-41.

[12] Khandekar RB, Tirumurthy S, Al-Harby S, et al. Diabetic retinopathy and ocular co-morbidities among persons with diabetes at Sumail Hospital of Oman. Diabetes Technology \& Therapeutics. 2009; 11(10): 675-9. https://doi.org/10.1089/dia.2009.0032

[13] Al-Futaisi A, Al-Zakwani I, Almahrezi A, et al. Prevalence and predictors of microalbuminuria in patients with type 2 diabetes mellitus: a cross-sectional observational study in Oman. Diabetes Research and Clinical Practice. 2006; 72(2): 212-5.

[14] Al-Moosa S, Allin S, Jemiai N, et al. Diabetes and urbanization in the Omani population: an analysis of a national survey data. Population Health Metrics. 2006; 4(5): 1-8. https ://doi .org/10.1186/14 78-7954-4-5

[15] Elliott JA, Abdulhadi NN, Al-Maniri AA, et al. Diabetes selfmanagement and education of people living with diabetes: a survey in primary health care in Muscat Oman. PLoS One. 2013; 8(2): 57400.

[16] Al-Sinani S, Al-Mamari A, Woodhouse N, et al. Quality of diabetes care at outpatient clinic, sultan qaboos university hospital. Oman Medical Journal. 2015; 30(1): 48-54. https ://doi .org/10.500 1/omj . 2015.09

[17] Alyaarubi S. Diabetes care in Oman: Obstacles and solutions. Sultan Qaboos University Medical Journal. 2011; 11(3): 343-48. PMid:22087375

[18] D'souza M, Venkatesaperumal R, Karkada S, et al. Determinants of glycosylated haemoglobin among adults with type 2 diabetes mellitus. Journal of Diabetes and Metabolism. 2013; 4(2): 265-71. https ://doi.org/10.4172/2155-6156.1000265

[19] Alrahbi H. Diabetes self-management (DSM) in Omani with type2 diabetes. International Journal of Nursing Sciences. 2014; 1(4): 352-9.

[20] Wellard SJ, Rennie S, King R. Perceptions of people with type 2 diabetes about self-management and the efficacy of community based services. Contemporary Nurse. 2008; 29(2): 218-26.

[21] Krueger RA, Casey MA. Focus groups: A practical guide for applied research. New Delhi: Sage; 2015.
[22] Polit DS, Beck CT. Nursing research: Principles and methods. 7th ed. Philadelphia: Lippincott Williams \& Willins; 2004.

[23] Morgan DL. Focus groups as qualitative research. Newbury Park, CA: Sage; 1996.

[24] Streubert HJ, Carpenter DR. Qualitative research in nursing: Advancing the humanistic imperative. 5th ed. Philadelphi: Lippincott Williams \& Wilkins; 2011.

[25] Miles MB, Huberman AM. Qualitative data analysis: A sourcebook. Beverly Hills: Sage; 1994.

[26] Doumato EA. Education in Saudi Arabia: gender, jobs, and the price of religion. In: Doumato EA, Posusney MP, editors. Women and Globalization in the Arab Middle East: Gender, Economy, and Society. London: Lynne Rienner; 2003. 239-58 p.

[27] Al Bimani ZS, Khan SA, David P. Evaluation of T2DM related knowledge and practices of Omani patients. Saudi Pharmaceutical Journal. 2015; 23(1): 22-7.

[28] Joo J, Lee H. Barriers to and facilitators of diabetes self-management with elderly Korean-American immigrants. International Nursing Review. 2016; 63(2): 277-84. https://doi.org/10.1111/jocn .12724

[29] Leung AYM, Bo A, Hsiao H, et al. Health literacy issues in the care of Chinese American immigrants with diabetes: A qualitative study. BMJ. 2014; 4(11): 1-12. https://doi.org/10.1136/bm jopen-2014-005294

[30] Carolan M, Holman J, Ferrari M. Experiences of diabetes selfmanagement: a focus group study among Australians with type 2 diabetes. Journal of Clinical Nursing. 2015; 24(7-8): 1011-23. https://doi.org/10.1111/jocn. 12724

[31] Mulcahy K, Maryniuk M, Peeples M, et al. Diabetes self-management education core outcomes measures. The Diabetes Educator. 2003; 29(5): 768-803. https://doi.org/10.1177/01457217030290 0509

[32] Weiler DM, Crist JD. Diabetes self-management in a Latino social environment. The Diabetes Educator. 2009; 35(2): 285-92. PMid:19204101 https://doi.org/10.1177/01457217083295 45

[33] Nunez MA, Yarandi H, Nunez-Smith M. Self-management among patients living with diabetes in the United States Virgin Islands. Journal of Health Care for the Poor and Underserved. 2011; 22(1): 271.

[34] Albright TL, Parchman M, Burge SK. Predictors of self-care behavior in adults with type 2 diabetes: an RRNeST study. Family Medicine. 2001; 33(5): 354-60. PMid:11355645

[35] Wilhelm K, Reddy J, Crawford J. The Importance of Screening for Mild Depression in Adults with Diabetes. Translational Biomedicine. 2017; 8(1): 101-8.

[36] Whittemore R, Melkus GDE, Sullivan A, et al. A nurse-coaching intervention for women with type 2 diabetes. The Diabetes Educator. 2004; 30(5): 795-804. PMid:15510531 https://doi.org/10.1 177/014572170403000515 